\title{
DISEÑO Y CONSTRUCCIÓN DE UNA RED DE FIBRA ÓPTICA PARA ANÁLISIS DE TOPOLOGÍAS Y TRANSMISIÓN DE SEÑALES EN DISPOSITIVOS PARA REDES WDM-PON
}

\author{
Claudia Milena Serpa ImbetT ${ }^{1}$ \\ Nelson Darío Gómez Cardona ${ }^{2}$ \\ Armando Borrero ${ }^{3}$ \\ Neil Guerrero GonZález ${ }^{4}$
}

\section{Resumen:}

En este trabajo se presenta el diseño e implementación de una red de fibra óptica en el laboratorio de fibras ópticas del Instituto Tecnológico Metropolitano que se configura en diferentes topologías y constituye un escenario con condiciones similares a las reales para realizar pruebas de dispositivos en enlaces de hasta $45 \mathrm{~km}$. Se presentan los resultados experimentales del análisis de pérdidas en diferentes tramos de la red, y de la transmisión de las señales provenientes de un multiplexor de fibra óptica de cuatro canales sobre 12

1 Ingeniera Física y Magíster en Ciencias Físicas, Universidad Nacional de Colombia - sede Medellín. Académica investigadora del Instituto Tecnológico Metropolitano. claudiaserpa@itm.edu.co.

2 Ingeniero Físico y Magíster en Ciencias Físicas, Universidad Nacional de Colombia - sede Medellín. Docente investigador del Instituto Tecnológico Metropolitano. nelsongomez@itm.edu.co

3 Docteur en Informatique, Université de Versalles Francia, Profesor Asociado de la Universidad de los Andes, Mérida Venezuela. Facultad de Ingeniería. e-mail: borrero@ula.ve.

4 Ingeniero Electrónico y Magíster en Automatización, Universidad Nacional de Colombia - sede Manizales. Profesor de la Universidad de Antioquia, estudiante de Doctorado de la Universidad Tecnológica de Dinamarca., Metro-Access and Short Range Systems Group, DTU Fotonik, Dinamarca. nggo@fotonik.dtu.dk 
km y $32 \mathrm{~km}$ en un escenario de una red óptica pasiva multiplexada por longitud de onda. Finalmente se implementa una topología en estrella y se analizan los resultados de potencia, pérdidas, y transmisión de pulsos de luz provenientes de un Reflectómetro Óptico en el dominio del tiempo. Este es el primer reporte en Colombia del desarrollo tecnológico de un ambiente en laboratorio con condiciones similares a las reales, con potenciales aplicaciones en las comunicaciones ópticas como desarrollos de sensores y enrutamiento óptico.

\section{Palabras claves:}

Red de fibra óptica, multiplexor óptico, topología, pérdidas

\section{Alostract:}

In this work, we designed and built a fiber optic-network in the optical laboratory of the Instituto Tecnológico Metropolitano. This network was used to emulate different topologies almost real conditions with the possibility to make connections up to $45 \mathrm{~km}$. We present experimental results of analysis in different network scenarios. Furthermore, we present experimental results for signal transmission and the transmission of four simultaneous channels by using an optics-multiplexor. Successful detection after 12 and $32 \mathrm{~km}$ through and passive optical network by wavelength division multiplexing is demonstrated. Finally, we present power and loss measures in terminals with a star topology and results for pulse transmission from Optical Reflectometer in the time domine. As far as we know, this is the first report and application in Colombia of a technological development of a laboratory network for topology analysis. This laboratory network will allow future applications like sensors testing and optical routing.

\section{Keywords:}

optical fiber network, optical multiplexor, topology, loss 


\section{INTRODUCCIÓN}

La incorporación de nuevos esquemas de transmisión en las redes de comunicaciones por fibra óptica está basado en el desarrollo de dispositivos recientes, topologías y nuevas arquitecturas, dando como resultado el incremento del ancho de banda y el uso eficiente del espectro para dar respuesta oportuna a los usuarios finales que cada día requieren de aplicaciones y servicios de alta velocidad (Al-Azzawi, 2007). En la actualidad, las nuevas tecnologías de transmisión basadas en multiplexación por longitud de onda (WDM, Wavelenght Division Multiplexing) en redes de fibra al hogar (FTTx, Fiber to the x), son una alternativa fuerte para responder a la necesidad inmediata de desarrollo e implementación de redes de alta capacidad que resistan la demanda y el volumen creciente de tráfico de internet (Sivalingan, 2000). Asimismo juegan un papel importante los esquemas de enrutamiento de las señales ópticas en los diferentes nodos de la red, y el diseño de arquitecturas y topologías adecuadas, en el incremento de la eficiencia de la transmisión, evitando retardos y enrutamientos no óptimos de la información (Dutta, 2004; Puerto, et al., 2008). En este sentido, debido a las condiciones particulares de adaptación de estas nuevas tecnologías en redes de transmisión, es deseable la evaluación del desempeño de los dispositivos, topologías y arquitecturas en un a mbiente similar al real, donde se evite la suspensión de servicios de las redes en funcionamiento, lo que constituye la propuesta de uso del diseño reportado en este trabajo.

Se presentan los resultados experimentales del análisis de pérdidas de una red de fibra óptica que se configura en distintas topologías, y que fue implementada en el laboratorio de fibras ópticas del Instituto Tecnológico Metropolitano (ITM), para prueba de dispositivos y en un enlace de hasta $45 \mathrm{~km}$ en un escenario con condiciones similares a las reales. Se analizan los resultados de la transmisión de las señales provenientes de un multiplexor (MUX) de fibra óptica de cuatro canales en $12 \mathrm{~km}$ y $32 \mathrm{~km}$ usando esta red en un escenario de una red óptica pasiva 
(PON, Passive Optical Network) multiplexada por longitud de onda WDM-PON. Finalmente se presentan la medición de potencia y pérdidas en las terminales de una topología tipo estrella, y los resultados de la transmisión de pulsos de luz provenientes de un Reflectometro Óptico en el Dominio del Tiempo (OTDR, Optical Time Reflectometer) en las distintas terminales usados en la caracterización de las fallas en la estrella.

Se propone esta construcción para la prestación de servicios de medición a los cableoperadores de Colombia en el laboratorio de fibras ópticas del ITM para verificación de dispositivos, análisis de topologías y transmisión de señales, con la ventaja de tiempo y espacio que ofrece un ambiente de laboratorio donde no es necesaria la suspensión de servicios de las redes en uso. Se propone en trabajos futuros el análisis de demultiplexores ópticos, otras topologías como bus y anillo, además de otros usos en transmisión de señales ópticas provenientes de sensores basados en sistemas distribuidos y multiplexados usando redes de Bragg en fibra óptica (FBG) y detección de fallas térmicas y deformaciones por reflectometría en enlaces largos (Kashyap, 1999; Grattan \& Sun, 2000).

\section{Montaje eXPerimental}

La Figura 1 muestra el montaje experimental el cual consiste de red modular NOYES construida con bobinas de $20 \mathrm{~km}$ de fibra óptica estándar monomodo (SMF) y $3 \mathrm{~km}$ de fibra óptica estándar multimodo (MMF). En UNO se extendió una bobina de $6 \mathrm{~km}$ de MMF constituida por cuatro hilos de 1,5 km de fibra unidos mediante empalmes por fusión realizados con una empalmadora Fujikura FS60; en DOS se extendió una bobina de 16 km de SMF de cuatro hilos de $4 \mathrm{~km}$, unidos de manera similar como en el caso anterior; en TRES se ubicó un divisor de potencia de 50/50 para obtener una terminal adicional. CUATRO es una posibilidad de interconexión interna con DOS o cualquier terminal. Esta construcción se usó para analizar la transmisión de señales provenientes de un multiplexor óptico (MUX) de fibra óptica construido con redes de Bragg (FBG) para las redes pasivas multiplexadas por longitud 
de onda (WDM-PON) bajo el esquema de la sección B de la Figura 1, que consiste en una fuente de luz LED Exalos EXS1560211 con un ancho espectral de $67 \mathrm{~nm}$ conectada a un aislador Newport ISC1550. La respuesta del MUX fue registrada usando un Analizador de Espectros Ópticos (OSA) de alta resolución Yokogawa AQ6370B, cuando sus señales fueron insertadas en $12 \mathrm{~km}$ de SMF (usando tres hilos de $4 \mathrm{~km}$ en la bobina de $16 \mathrm{~km}$ ) desde T2 hasta DOS, y $32 \mathrm{~km}$ de SMF desde T2 hasta TRES. El esquema de la sección C se hizo extensiones en $\mathrm{T} 1$ y T2 usando divisores de potencia de 5/95 y 67/33 para obtener una estrella de cuatro terminales rotuladas como A, B, C y D. En DOS se ubicó una fuente de luz EXFO FLS60 y en $\mathrm{A}, \mathrm{B}, \mathrm{C}, \mathrm{D}$ se registraron los niveles de potencia usando un detector EXFO PM60. Posteriormente, Con el OTDR EXFO 7500 se realizaron registros desde A, B, C y D hasta DOS.

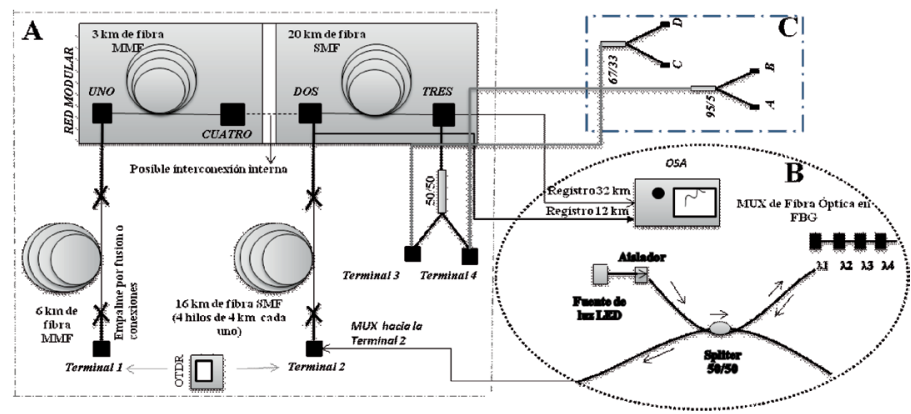

Figura 1. Montaje experimental de La Red, MUX óptico, y topología estrella

\section{Resultados eXPERIMENTALES}

Se usó el OTDR EXFO 7500, para verificar la continuidad entre las Terminales y la red modular bajo diferentes esquemas de conexión, mediante la evaluación de las pérdidas y la reflectividad. Se realizaron pruebas en la Terminal 1(T1) y la Terminal 2 (T2). La Figura 2 muestra el registro de la T1 hasta DOS y de T2 hasta TRES usando el OTDR con longitudes de onda de $1310 \mathrm{~nm}$ y 1550 $\mathrm{nm}, \mathrm{y}$ un ancho de pulso de $1 \mu \mathrm{s}$. La Figura 2 a muestra el registro de T1 hasta DOS a $1550 \mathrm{~nm}$, donde la atenuación promedio es de 
$0.582 \mathrm{~dB} / \mathrm{km}$ y la pérdida máxima por empalme de $0.425 \mathrm{~dB}$. La pérdida total en este segmento de $\sim 9,2 \mathrm{~km}$ es de $5.409 \mathrm{~dB}$. Los eventos reflexivos representados por "picos" están ubicados a $\sim 6.2$, 7.6, y $8.7 \mathrm{~km}$ y registran una reflectividad de $-59,3,-48,1,-43,8 \mathrm{~dB}$ respectivamente donde $-43,8 \mathrm{~dB}$ corresponde al "pico" más alto en el enlace. Para el registro a $1310 \mathrm{~nm}$ la atenuación promedio es de $0.904 \mathrm{~dB} / \mathrm{km}$ y la pérdida de $8,403 \mathrm{~dB}$. La variación de estos valores con respecto a los registrados a $1550 \mathrm{~nm}$ es debido a la dependencia de la atenuación y las pérdidas con la longitud de onda del pulso de prueba (Al-Azzawi, 2007). A diferencia de los valores de pérdida y atenuación, la reflectividad es similar a ambas longitudes de onda debido a que no hay una dependencia de este parámetro con la misma. Similarmente, la Figura $2 \mathrm{~b}$ muestra el registro desde la Terminal 2 hasta TRES. Para el registro a $1550 \mathrm{~nm}$, la atenuación promedio y la pérdida total en este segmento de $36 \mathrm{~km}$ es de 0.369 $\mathrm{dB} / \mathrm{km}$ y 13,33 dB. Los eventos reflexivos están ubicados a 16, 17, 23,31 y $32 \mathrm{~km}$ y registran una reflectividad que va desde $23,5 \mathrm{~dB}$ para los hasta 60,1 dB. A $1310 \mathrm{~nm}$ la atenuación promedio es de $0.541 \mathrm{~dB} / \mathrm{km}$ y la pérdida de $19,478 \mathrm{~dB}$. La Terminal 4 presenta pérdida por inserción de $\sim 1,5 \mathrm{~dB}$ según los datos del fabricante.
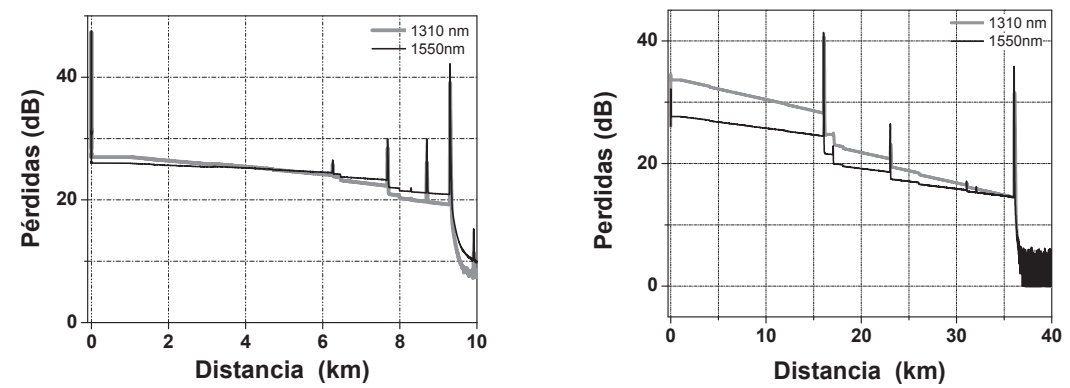

Figura 2. A) Registro desde T1 hasta dOS, b) Registro desde la t2 hasta TRES

La Figura 3a muestra la respuesta de un MUX de fibra óptica de cuatro canales cuando las señales fueron transmitidas a través de $12 \mathrm{~km}$ de fibra SMF. Las potencias máximas medidas fueron aproximadamente $\sim-42 \mathrm{dBm}$ para cada canal. Las pérdidas por 
canal son aproxiadamente $\sim 2 \mathrm{~dB}$, con respecto a la potencia inicial en el MUX.

En la Figura 3b se observa el desempeño para una transmisión sobre $32 \mathrm{~km}$ de fibra SMF donde la potencia máxima para cada canal es de $\sim-72 \mathrm{dBm}$, dando como resultado una pérdida por cada longitud de onda de aproximadamente $\sim 30 \mathrm{~dB}$.
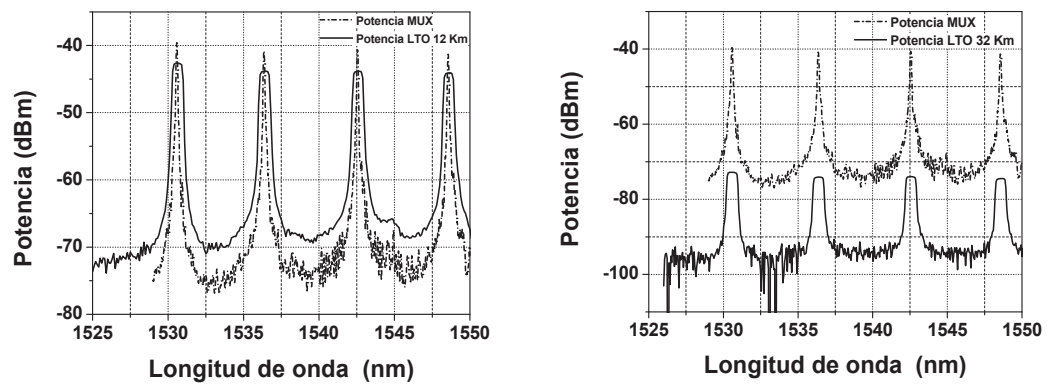

Figura 3. RESPUESTA DE UN MULTIPLEXOR ÓPTICO CUANDO LAS SEÑALES SON INSERTADAS EN A) 12 Y B) 32 KM DE FIBRA RESPECTIVAMENTE

Los resultados de la configuración en estrella muestran que la potencia inicial de la fuente de luz FLS60 fue de $+2,08$ y -2,25 dBm. En la Figura 4 se muestra la potencia y las pérdidas registrada en las terminales con respecto a la relación de división de cada acoplador (Snyder \& Love, 2000).
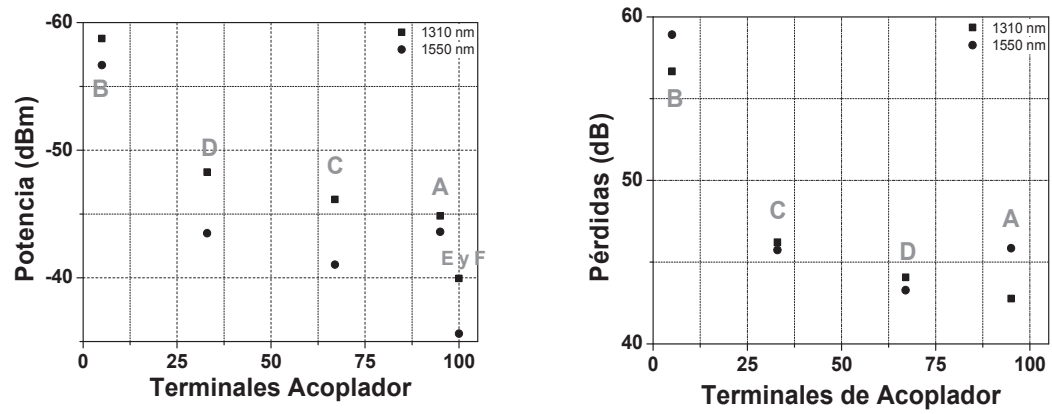

Figura 4. A) Potencia Óptica en las terminales del los acopladores a $1310 \mathrm{Y}$ 1550 nM, B) PÉrdidas en las terminales de los acopladores a 1310 y 1550 NM 
En la Figura 5 se muestran los resultados experimentales en cada terminal obtenidos con el OTDR con un ancho de pulso de $275 \mathrm{~ns}$ a $1310 \mathrm{~nm}$ y $1550 \mathrm{~nm}$ respectivamente, con el registro en A se reporta la detección de 5 fallas a $\sim 0,2,10,13,18,19 \mathrm{~km}$, con el registro en $\mathrm{B}$ se reporta la detección de 2 fallas a $\sim 0,01$ y 0,05 $\mathrm{km}$, se observa que el alcance máximo del pulso es de $160 \mathrm{~m}$, con el registro en $\mathrm{C}$ se reporta la detección de 5 fallas a las mismas distancias de la parte a) y con el registro en $\mathrm{D}$ se reporta la detección de 4 fallas a 5, 10, 13, $19 \mathrm{~km}$. En general, se muestra que las topologías en A y $\mathrm{C}$ son las que configuran caminos óptimos para la detección de fallas.
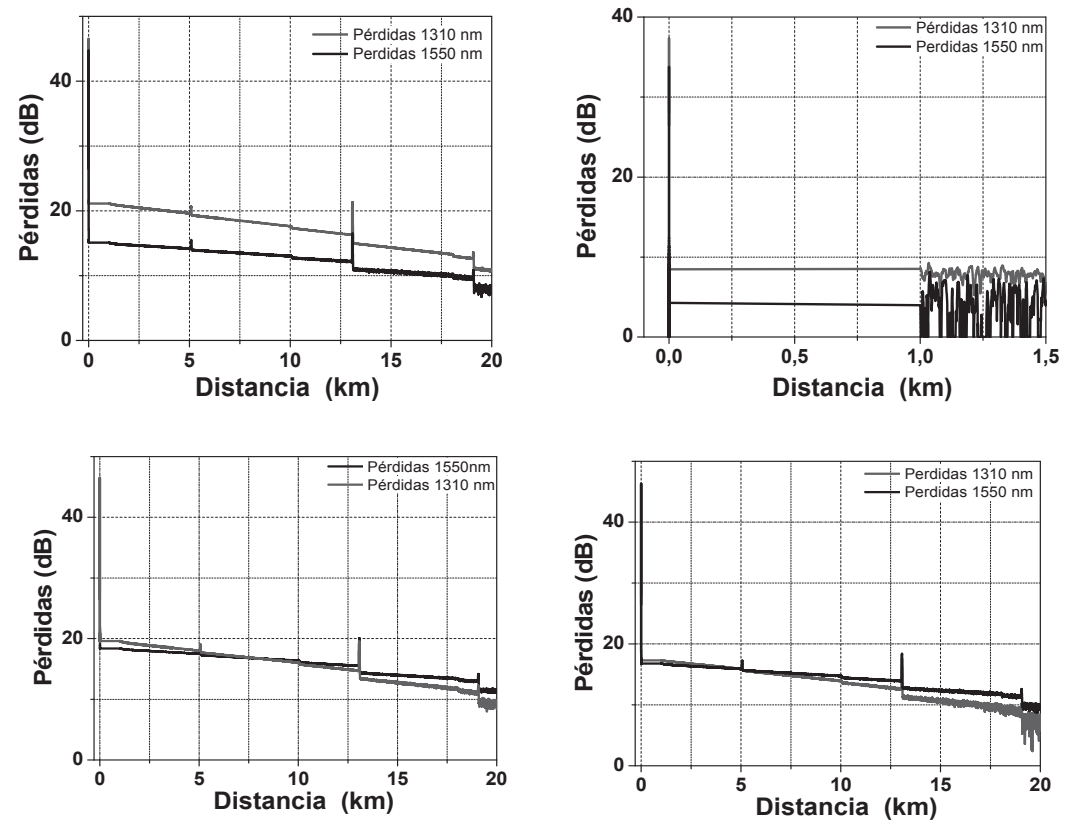

Figura 5. a) Registro en A, b) Registro en B, c) Registro en C y d) Registro en D

\section{Discusión}

Se verificó el funcionamiento de los diferentes segmentos de la configuración reportada en este trabajo, a través del análisis 
de la atenuación promedio en el enlace, la pérdida promedio, y la reflectividad. Se comprobó que la atenuación promedio y pérdidas registran valores diferentes a $1550 \mathrm{~nm}$ y $1310 \mathrm{~nm}$, y que los valores de reflectividad son similares para las dos longitudes de onda. Esta configuración posibilita la simulación de diferentes topologías y distancias, así como la ampliación de la misma por adición de terminales con el uso de divisores, multiplexores y demultiplexores (Sivalingam, 2005).

La transmisión de las señales provenientes de un MUX después de $32 \mathrm{~km}$ registra una pérdida de aproximadamente $30 \mathrm{~dB}$ medida con el OTDR. Se concluye que existe un error de $23 \%$ entre las pérdidas del MUX obtenidas a través de registros espectrales usando el OSA y las obtenidas con el OTDR. Las longitudes de onda de las señales del MUX están alrededor de 1532, 1536, 1543 y $1548 \mathrm{~nm}$ y en este caso las pérdidas a estas longitudes de onda son mayores que las registradas a $1550 \mathrm{~nm}$. Esto se debe a la relación inversa entre las pérdidas y longitudes de onda. (Agrawal, 2007).

Se construyó una terminal tipo estrella usando acopladores con diferentes razones de acoplamiento: (50/50, 5/95, 33/67). En la Figura 4 se observa una dependencia exponencial de las pérdidas y la potencia, y los resultados experimentales obtenidos se ajustan a funciones exponenciales decrecientes (Snyder \& Love, 2000). Los resultados muestran que en la terminal A y C la detección de fallos fue de cinco, en el punto D de cuatro y en el punto B de dos, mostrando que los caminos más adecuados para detección de fallas son desde las terminales A y C hasta el enlace. En la terminal B se detectan dos eventos debido a la poca potencia que viaja hacia el enlace (solo del $5 \%$ de la inicial)

\section{Conclusiones}

La red construida se propone para la prueba de dispositivos en diferentes configuraciones y topologías. En este trabajo se reportó el desempeño de un MUX en una de las posibles configuraciones de esta red, y la posibilidad de analizar configuraciones en estrella 
para determinar los caminos óptimos para caracterización de enlaces desde las terminales de la estrella hasta la red. En la red construida es posible realizar análisis de transmisión de dispositivos como demultiplexores ópticos, otras topologías como anillo y bus, además de análisis de sistemas de sensado por fibra óptica.

\section{Agradecimientos}

Este trabajo hizo con fondos del Centro de Investigación del Instituto Tecnológico Metropolitano de Medellín-Colombia, en el proyecto Código: P07203. A la empresa Exalos S.A de Zurich Suiza por la donación de la fuente de luz SLED Exalos EXS1560211 usada en la construcción del MUX de fibra óptica.

\section{RefERencias}

Agrawal, G. (2007). Fiber-optic communication systems. Michigan: WileyInterscience.

Al-Azzawi, A. (2007). Fiber optics: principles and practices: Optical Science And Engineering Series. Boca Raton: Optical Science And Engineering Series.

Dutta, N. K. (2004). WDM technologies: Optical Network. New York: Academic Press.

Grattan, K., \& Sun, T. (2000). Fiber Optic Sensor Technology: an Overview. Sensor and Actuators , 40-61.

Kashyap, R. (1999). Fiber Bragg gratings Optics and photonics.

Puerto, G., Ortega, B., Capmany, J., Cardona, K. \& Suarez, C. (2008). Evolución de las redes de datos: Hacia una plataforma de comunicaciones completamente óptica. Revista Facultad de Ingeniería Universidad de Antioquia, 148-158.

Sivalingan, K. (2000). Optical WDM networks: principles and practice. New York: Springer.

Sivalingam, K. (2005). Emerging optical network technologies: architectures, protocols, and performance. Washington: Springer.

Snyder, A. \& Love, J. (2000). Optical Waveguide Theory. Norwell: Kluwer Academic Publisher. 